\title{
Sentido, referencia y representación lingüística en Frege
}

\author{
LUIS FERNÁNDEZ MORENO ${ }^{1}$ \\ Universidad Complutense de Madrid
}

\section{INTRODUCCIÓN}

FREGE EMPLEÓ EL TÉRMINO "representación" ("Vorstellung") para aludir a un tipo de entidades de carácter mental, como la imagen mental que tenemos de un objeto del mundo externo, e hizo hincapié en el carácter subjetivo de tales entidades (véase 1892a, pp. 29-31, así como 1918, pp. 66 s.). Frege apeló a la noción de representación para, por contraposición con ella, permitir una mejor comprensión de las nociones de sentido y de referencia -especialmente de la primera de ellas- (en 1892a) y de la noción de pensamiento, es decir, del sentido de las oraciones o de los enunciados ${ }^{2}$ (en 1918). En este artículo no entenderé el término "representación" en el sentido fregeano del término, sino en una acepción hoy más usual, según la cual distintos tipos de entidades linguísticas poseen un carácter representacional y son, por tanto, representaciones. Esta acepción del término se encuentra, por ejemplo, en Wittgenstein (1922). Este autor formula una teoría de la figuración o representación, según la cual las oraciones (con sentido) son concebidas como un tipo de figuras o representaciones, siendo lo representado por ellas estados de cosas, ya sean existentes -hechos- o no-existentes (1922, proposición 2.11). En la obra de Frege las oraciones poseen también un carácter representacional, si bien a este respecto él no empleó, por las razones indicadas, el término "representación".

El objetivo de este artículo es explicitar y examinar la teoría de la representación lingüística contenida en la obra de Frege, si bien limitaremos nuestras consideraciones a uno de los tipos de enunciados más simples, a saber, aquellos que constan de un nombre propio y de un predicado monádico o término

1 Dpto. de Lógica y Filosofía de la Ciencia, Facultad de Filosofía, Universidad Complutense de Madrid, Ciudad Universitaria, 28040 Madrid, e-mail: luis.fernandez@ filos.ucm.es.

2 En este trabajo emplearé de manera intercambiable las expresiones "oración" -en el sentido de oración declarativa- y "enunciado". 
conceptual; ${ }^{3}$ no obstante, las observaciones que hagamos acerca de este tipo de enunciados se extenderían, con las modificaciones correspondientes, al resto de los enunciados. Ahora bien, la teoría de la representación lingüística que cabe atribuir a Frege descansa en la noción de referencia, la cual a su vez presupone la noción de sentido; por este motivo habremos de ocuparnos en detalle de la teoría de Frege acerca del sentido y de la referencia de las expresiones componentes de dichos enunciados. Puesto que lo representado por esos enunciados serán entidades o relaciones entre entidades, conviene que comencemos introduciendo algunas de las nociones básicas de la ontología y de la semántica de Frege.

\section{ONTOLOGÍA, SEMÁNTICA Y REPRESENTACIÓN}

Como es sabido, Frege distingue dos tipos de expresiones, saturadas y no-saturadas; a esta división de las expresiones le corresponde la división de carácter ontológico en entidades saturadas u objetos y entidades no-saturadas o funciones. Las funciones son designadas mediante las expresiones no-saturadas, a las que Frege también alude como "expresiones de función", "nombres de función" o "signos de función", entre las que se encuentran los predicados o términos generales (véase la nota 2). Por su parte, los objetos son designados mediante las expresiones saturadas, a las que Frege denomina nombres propios. De esta manera Frege entiende la noción de nombre propio en un sentido muy amplio, bajo la cual quedan subsumidos no sólo los nombres propios en el sentido usual del término, sino también las descripciones definidas (singulares) e incluso las oraciones. La consideración de las oraciones como un tipo de nombres propios -en la acepción fregeana del término-, es decir, como un tipo de expresiones saturadas es comprensible si se toma en consideración que las expresiones no-saturadas pueden ser complementadas mediante expresiones saturadas para dar lugar a otras expresiones saturadas, y justamente las oraciones pertenecerán a las expresiones saturadas resultantes de la complementación de expresiones no-saturadas por parte de expresiones saturadas más simples; así, por ejemplo, un predicado monádico puede ser saturado mediante un nombre propio, en el sentido usual del término, ${ }^{4}$ o por una descripción definida.

3 Los términos conceptuales constituyen uno de los tipos de términos generales más importantes en la obra de Frege -su caracterización será presentada dos párrafos más abajo. Empleo las expresiones "predicado monádico" y "término conceptual" de manera indistinta, aunque en muchos casos cabe distinguir entre un predicado monádico y el término conceptual con el que se ha formado el predicado. La misma consideración se aplica, a nivel más general, a la relación entre predicados y términos generales.

4 A partir de ahora entenderé por "nombre propio" sólo los nombres propios en el sentido usual del término, a los que Frege aludió en ocasiones como "auténticos 
De manera pareja, esta vez a nivel ontológico, las funciones pueden ser complementadas mediante objetos, que serán los argumentos de la función, para dar lugar a otros objetos, a saber, a los valores de la función para dichos argumentos. Entre estos valores y, por tanto, entre los objetos se encuentran los valores de verdad, a los que Frege denomina "lo verdadero" y "lo falso" y que, según él, son las entidades designadas por las oraciones. Un tipo muy importante de funciones son los conceptos, es decir, las funciones de un único argumento cuyo valor es un valor veritativo. Las expresiones lingüísticas que designan conceptos son un tipo de términos generales a los que Frege denomina términos conceptuales. De este modo uno de los tipos de oraciones más simples, y las únicas que tomaremos en consideración en lo siguiente, constarán de un nombre propio y de un término conceptual y, puesto que los nombres propios designan objetos y los términos conceptuales designan conceptos, lo representado por una oración verdadera será una relación entre un objeto y un concepto, a saber, la relación consistente en que un objeto cae bajo un concepto; en este caso el objeto pertenece al conjunto que constituye la extensión del concepto y el valor del concepto para ese objeto es lo verdadero. En el caso de una oración falsa lo representado será igualmente una relación entre un objeto y un concepto, pero aunque la oración representa el objeto como estando en la relación de caer bajo ese concepto, esta relación no se da en el mundo real, por lo que el objeto no pertenece al conjunto que constituye la extensión del concepto y el valor del concepto para ese objeto es lo falso. Otra forma de expresar lo representado por un enunciado simple del tipo mencionado consiste en apelar a la noción de propiedad. Para Frege un concepto es una propiedad de los objetos que caen bajo el concepto. Por tanto, lo representado mediante ese tipo de enunciados será la atribución de una propiedad a un objeto; en el caso de un enunciado verdadero, el objeto en cuestión tiene la propiedad que se le atribuye, mientras que no ocurre así en el caso de un enunciado falso. En definitiva, lo representado por las oraciones que constan de un nombre propio y de un término conceptual son relaciones, efectivas o meramente supuestas, entre objetos y conceptos o propiedades.

Ahora bien, en la obra de Frege los enunciados no constituyen el único tipo de entidad susceptible de llevar a cabo la función de representación, pues también cumplen dicha función los sentidos de los enunciados, es decir, los pensamientos; por este motivo hemos de tomar en consideración la noción de sentido.

Frege introduce la noción de sentido en (1892a) a la hora de explicar el distinto valor cognoscitivo de los enunciados de identidad (verdaderos) del tipo

nombres propios" -véase el texto de Frege sobre el nombre propio "Aristóteles" citado más abajo. 
" $\mathrm{a}=\mathrm{b}$ " con respecto a los del tipo " $\mathrm{a}=\mathrm{a}$ " - "a" y " $\mathrm{b}$ " sustituyen a nombres propios-, pues los enunciados de identidad del primer tipo, a diferencia de los del segundo, pueden aumentar nuestro conocimiento. Frege explica esta diferencia apelando a que, aunque los nombres "a" y "b" poseen la misma referencia, tienen, sin embargo, distinto sentido. De esta manera el sentido de un nombre propio es una dimensión del nombre que contribuye al valor cognoscitivo de los enunciados en los que figura. La noción de sentido es así una noción de carácter epistémico; el sentido de una expresión es un rasgo de la expresión que conocemos cuando la entendemos. Pero la noción de sentido es asimismo una noción de carácter semántico, pues el sentido de una expresión determina su referencia ${ }^{5}$ o, como suele decirse, es el modo como la expresión nos presenta su referente. Así, y sirviéndonos de uno de los ejemplos más famosos de Frege, las expresiones "lucero vespertino" y "lucero matutino" poseen la misma referencia, puesto que ambas designan el planeta Venus, pero nos presentan su referente de distinta manera, a saber, respectivamente como el astro que más brilla al atardecer y como el astro que más brilla al amanecer. En su vertiente semántica el sentido de una expresión puede caracterizarse como una condición para identificar su referente; el referente de una expresión será la entidad que satisfaga su sentido.

Como ya hemos indicado, el sentido de un enunciado es un pensamiento y la referencia de un enunciado es su valor de verdad, si bien los portadores primarios de verdad no son, según Frege, los enunciados, sino los pensamientos: una oración es verdadera si y sólo si el pensamiento expresado por ella es verdadero. Esta tesis acerca de la prioridad de los pensamientos sobre los enunciados como portadores de verdad se extiende también a su carácter representacional; lo representado por un enunciado es lo representado por el pensamiento expresado por él, y de la misma manera que en un enunciado simple del tipo que estamos tomando en consideración cabe distinguir entre una expresión saturada, es decir, un nombre propio, y una expresión no-saturada, es decir, un término conceptual, en el pensamiento, que es, al igual que el enunciado que lo expresa, una entidad saturada, cabe distinguir entre un componente saturado y uno no-saturado, siendo éstos respectivamente el sentido de la expresión saturada y de la expresión no-saturada componentes del enunciado. Y puesto que la referencia de un nombre propio es un objeto y la de un término conceptual un concepto, al componente saturado del pensamiento le corresponde un objeto

5 Como ocurre en la obra de Frege, emplearé el término "referencia" de manera ambigua, a saber, para aludir tanto a lo designado por una expresión -el referente de la expresión- como a la relación entre una expresión lingüística y lo designado por ella; aunque el contexto aclarará la ambigüedad en el uso de este término, cuando emplee el término "referencia" en la primera acepción alternaré su uso en ocasiones con el término "referente". 
y al no-saturado, un concepto. Lo representado por los pensamientos expresados por los enunciados del tipo mencionado son, como lo representado por estos enunciados, relaciones, efectivas o meramente supuestas, entre objetos y conceptos o propiedades. Pero la circunstancia de que un objeto caiga bajo un concepto o de que un objeto tenga una propiedad no constituye un hecho, pues para Frege los hechos pertenecen al ámbito del sentido, no de la referencia: "Un hecho es un pensamiento verdadero" (Frege 1918, p. 50). Por tanto, en la teoría de la representación y, en concreto, de la representación lingüística que cabe atribuir a Frege las entidades representadas no son hechos, sino relaciones entre entidades pertenecientes al ámbito de la referencia.

A pesar de que en la teoría de Frege la representación tiene lugar de manera primaria al nivel de los pensamientos, en este artículo vamos a centrarnos fundamentalmente en su teoría de la representación lingüística, es decir, de la representación mediante enunciados y, más específicamente, mediante los enunciados simples que constan de un nombre propio y de un término conceptual. Puesto que estos enunciados representan una relación entre la referencia de un nombre propio y la de un término conceptual, habremos de atender con detenimiento a la teoría de la referencia de los nombres propios y de los términos conceptuales formulada por Frege, pero también a la teoría acerca de su sentido, ya que, por una parte, el sentido de una expresión determina su referencia y, por otra, los sentidos de esos dos tipos de expresiones son los componentes de los pensamientos expresados por dichos enunciados, de los que deriva el carácter representacional de tales enunciados. Ahora bien, a este respecto conviene señalar que Frege se ocupó principalmente del sentido de los nombres propios, pues lo afirmado por él acerca del sentido de los términos conceptuales posee un carácter fragmentario; el escrito que pretendía ser su contribución fundamental a esta temática lo constituye un manuscrito posiblemente incompleto, publicado póstumamente, a saber, Frege (1892-1895).

\section{EL SENTIDO Y LA REFERENCIA DE LOS NOMBRES PROPIOS ${ }^{6}$}

Como hemos indicado, la referencia de un nombre propio es un objeto, $\mathrm{y}$ esta referencia viene determinada $\mathrm{y}$, por tanto, mediada por el sentido del nombre. En (1892a) Frege afirma que "[e]l sentido de un nombre propio lo comprende todo aquel que conoce el lenguaje o el conjunto de designaciones al que pertenece" (1892a, p. 27); en esta afirmación, en la que Frege está tomando en consideración los lenguajes naturales y no sólo los lenguajes (lógicamente) perfectos -o supuestamente tales, como el ideado por Frege, la conceptografía-, 
se habla de "el sentido de un nombre propio", como si cada nombre propio tuviese un único sentido, de tal manera que todo hablante competente en el uso de un nombre propio habría de saber cuál es el sentido del nombre. Pero tras esta afirmación Frege añade una famosa nota a pie de página a la que es imprescindible atender:

En el caso de un auténtico nombre propio como ‘Aristóteles' naturalmente pueden dividirse las opiniones en cuanto a su sentido. Por ejemplo, se podría suponer que este sentido es: el discípulo de Platón y maestro de Alejandro Magno. Quien suponga esto, atribuirá al enunciado 'Aristóteles era originario de Estagira' un sentido distinto de aquel para quien el sentido de este nombre fuese: el maestro de Alejandro Magno originario de Estagira. Mientras la referencia siga siendo la misma pueden tolerarse estas oscilaciones del sentido, a pesar de que deben evitarse en el edificio conceptual de una ciencia demostrativa y de que no deberían aparecer en un lenguaje perfecto. (Frege 1892a, p. 27, n. 2).

En este pasaje Frege reconoce que distintos hablantes competentes en el uso de un nombre propio pueden asociar con él sentidos distintos por cuanto pueden asociar con el nombre descripciones definidas distintas; de esta manera no hay, por regla general, una única descripción que exprese el sentido de un nombre propio. La misma idea aparece formulada en (1918), en relación con el nombre "Dr. Gustav Lauben". Allí Frege alude a dos hablantes, Herbert Gauner y Leo Peter, que asocian con este nombre un sentido diferente; uno de esos sentidos vendría expresado por la descripción "el único individuo nacido el 13 de septiembre de 1875 en N.N.", mientras que el otro vendría expresado mediante una descripción similar a "el único médico que vive en tal y tal vivienda" (1918, pp. 65 s.).

Ahora bien, las afirmaciones de Frege en las que éste indica que el sentido de un nombre propio puede ser diferente para hablantes diferentes entran en conflicto con la afirmación mencionada anteriormente de que "[e]1 sentido de un nombre propio lo comprende todo aquel que conoce el lenguaje o el conjunto de designaciones al que pertenece" (1892a, p. 27), lo que sugiere que cada nombre propio tiene un único sentido para todos los hablantes de un mismo lenguaje. De esta manera en la obra de Frege hay una tensión entre la posición de que cada nombre propio posee, al menos por regla general, un único sentido, que es compartido por todos los hablantes competentes en el uso del nombre propio y que, por tanto, tiene un carácter público, y la posición de que en los lenguajes naturales, por regla general, no cabe hablar de "el sentido de un nombre propio", sino de su sentido para un hablante determinado, con lo que el sentido de un nombre propio, aunque podría ser compartido, de hecho no lo será y, por tanto, no cabe sostener que el sentido de un nombre propio 
es público, ya que distintos hablantes podrán asociar con un nombre propio sentidos diferentes.

No obstante, en el pasaje mencionado Frege considera que las diferencias correspondientes a los distintos sentidos asociados con un nombre propio en los lenguajes naturales son admisibles en la medida en que esos sentidos determinen la misma referencia. Esta afirmación es coherente con la teoría de la representación contenida en la obra de Frege, pues a este respecto lo relevante son la referencias de las expresiones y, aunque pensamientos diferentes constituyan diferentes representaciones, lo representado por ellos puede ser lo mismo. Ahora bien, puesto que los pensamientos expresados mediante enunciados emitidos por hablantes que asocien con un nombre propio sentidos diferentes son pensamientos diferentes, pueden plantearse problemas de comunicación entre esos hablantes. A este respecto Frege afirma en (1918) en relación con el ejemplo del nombre "Dr. Gustav Lauben", al que ya hemos aludido, que dos hablantes que asocian con un nombre propio sentidos diferentes, aunque éstos determinen la misma referencia, no hablan en relación con ese nombre el mismo lenguaje (1918, p. 67). Además, el carácter informativo de un enunciado se verá influido por el sentido asociado con sus expresiones componentes, por lo que si aceptamos que distintos hablantes asocian - o pueden asociar-con un nombre propio sentidos diferentes, podrá ocurrir que un enunciado en el que figure un nombre propio sea informativo para unos hablantes y no para otros; así, por ejemplo, el enunciado "Aristóteles era originario de Estagira" no será informativo para el hablante considerado en segundo lugar en el pasaje de Frege acerca del nombre "Aristóteles", mientras que podrá serlo para el hablante contemplado en primer lugar en dicho pasaje.

Para concluir nuestras consideraciones sobre el pasaje mencionado, cabe señalar que en "un lenguaje perfecto", como el supuestamente ideado por Frege, cada nombre propio y, en general, toda expresión habría de tener un único sentido (para todo hablante) y, como se indicó anteriormente, una (única) referencia. Pero la primera condición parece ser requerida por Frege incluso para los lenguajes naturales:

[E]n un nombre propio importa cómo se da a través de él [...] lo designado. Esto puede suceder de modos diferentes y a cada uno de ellos le corresponde un sentido particular de una oración que contiene el nombre propio. Los diferentes pensamientos que resultan de la misma oración coinciden, ciertamente, en su valor de verdad [...] Sin embargo, ha de admitirse su diversidad. Por tanto, ha de estipularse que con cada nombre propio esté vinculado un único modo de darse [...] lo designado por él. (Frege 1918, pp. 65-66).

En este texto se reconoce que con un nombre propio puede haber asociados diferentes sentidos, de lo que se sigue que una oración que contenga un 
nombre propio podrá expresar diferentes pensamientos y, por tanto, constituir diferentes representaciones, proponiéndose como remedio a dicha situación la estipulación para cada nombre de un único sentido. Ahora bien, el llevar a cabo estipulaciones de este tipo puede ser admisible para lenguajes artificiales, como la conceptografía, pero es irreal suponer que dichas estipulaciones han de llevarse a cabo con respecto a los lenguajes naturales: quién establecería tales estipulaciones y con qué derecho.

En cualquier caso, y tras haber constatado que Frege reconoce que distintos hablantes pueden asociar con un nombre propio sentidos distintos, podemos dejar de lado esta cuestión para ocuparnos de otra que nos es más importante, a saber, la cuestión de cómo viene determinada la referencia de un nombre propio. Como ya indicamos, el sentido de una expresión determina su referencia; ahora bien, y por lo que concierne a los nombres propios, en el pasaje citado acerca del nombre propio "Aristóteles" Frege parece sostener que el sentido de un nombre propio vendría expresado mediante una descripción definida y la justificación de esta afirmación radicará en que el sentido del nombre propio será idéntico al sentido de una descripción definida; la conclusión a extraer es que la referencia de un nombre propio viene determinada por (el sentido de) una descripción definida, a saber, por la descripción definida que expresa el sentido del nombre propio. Por este motivo no es de extrañar que la teoría de la referencia de los nombres propios que suele atribuírsele a Frege sea la siguiente: la referencia de un nombre propio viene determinada mediante una descripción definida -ésta será la descripción definida que el hablante asocia con el nombre. Como hemos indicado, esa conclusión se sigue de dos tesis. Primera, la referencia de un nombre propio viene determinada por su sentido. Segunda, el sentido de un nombre propio es idéntico al sentido de una descripción definida $-\mathrm{y}$, por tanto, el sentido del nombre es expresado mediante una descripción definida. Con respecto a esta segunda tesis conviene hacer al menos tres observaciones.

En primer lugar, cabe preguntarse cuándo dos expresiones poseen el mismo sentido. En (1892a) Frege propone el siguiente criterio de identidad de pensamientos: dos pensamientos son idénticos si nadie que los capte puede considerar que tienen distinto valor de verdad, es decir, que uno de ellos sea verdadero y el otro falso (1892a, p. 32; véase también 1983, p. 213 y 1976, pp. 105 s. y 128). Este criterio trae consigo el siguiente criterio de identidad de sentidos: dos sentidos son idénticos si nadie que los capte puede considerar que les corresponde una referencia diferente. Por tanto, dos expresiones -con referencia- poseen el mismo sentido si ningún hablante que las entienda puede dejar de saber que tienen la misma referencia y dos expresiones con la misma referencia poseen un sentido distinto si es posible entenderlas sin saber que tienen 
la misma referencia. ${ }^{7}$ Ahora bien, si tomamos en consideración las expresiones "Aristóteles" y "El discípulo de Platón y maestro de Alejandro Magno", no parece haber óbice para que haya hablantes que las entiendan pero puedan considerar que tienen una referencia diferente. Más aún, incluso sabiendo que esas dos expresiones designan, de hecho, el mismo individuo, habrá de reconocerse que en situaciones contrafácticas podrían designar un individuo diferente. ${ }^{8}$ Por tanto, la tesis de que el sentido de un nombre es idéntico al de una descripción definida y viene expresado por ella es sumamente cuestionable, a menos que la descripción definida expresase una propiedad necesaria del referente del nombre. Por tanto, por regla general, la segunda tesis es falsa.

En segundo lugar, y trayendo a colación la primera tesis, cabe señalar que la referencia de un nombre propio viene determinada por su sentido porque el sentido de un nombre proporciona una condición para identificar el referente del nombre. Ahora bien, aunque una descripción definida pueda expresar una condición para identificar el referente de un nombre, ya que éste será el (único) objeto que satisfaga la descripción definida, de aquí no se sigue, como ya hemos indicado, que el nombre y la descripción definida hayan de poseer el mismo sentido. La descripción definida puede proporcionarnos una condición que identifique de hecho el referente del nombre, pero podríamos imaginar situaciones contrafácticas en las que esto no fuese así.

En tercer lugar, de la tesis de que el sentido de un nombre es idéntico al sentido de una descripción definida se sigue que ésta expresa el sentido del nombre. Pero la afirmación de que el sentido de un nombre viene expresado mediante una descripción definida conlleva otras consecuencias inaceptables, además de las ya indicadas. Si el sentido de un nombre propio viene expresado mediante una descripción definida que, a su vez, contiene nombres propios -como ocurre en el ejemplo de Frege acerca del nombre "Aristóteles"-, podríamos vernos involucrados en un círculo vicioso o en un regreso al infinito. En un círculo vicioso, en caso de que el sentido del nombre contenido en la descripción definida viniese expresado, a su vez, por alguna descripción definida en la que figurase el primer nombre; mientras que de no ser así, podríamos vernos involucrados en un regreso al infinito. Pero si la determinación de la referencia de un nombre nos involucra en un círculo vicioso o en un regreso al infinito, la referencia del nombre nunca podría quedar determinada. Una manera de

$7 \quad$ El criterio de identidad de sentidos propuesto por Frege contiene una condición que lo hace mucho más fuerte, pues Frege afirma que la identidad del sentido de expresiones ha de ser reconocida con evidencia de manera inmediata (véase 1983, pp. 226 s.). En lo siguiente pasaré por alto esta condición, pues no es esencial para la cuestión que nos ocupa.

8 Argumentos de este tipo han sido formulados de manera paradigmática por Kripke en $(1980)$. 
evitar el vernos abocados a tal situación consistiría en apelar a descripciones definidas que sólo contuviesen términos puramente generales, es decir, términos generales que expresen propiedades que no involucren individuos específicos, pero es inverosímil que un individuo pueda ser singularizado exclusivamente mediante tal tipo de propiedades.

De acuerdo con las consideraciones precedentes, la posición de Frege acerca de la determinación de la referencia de los nombres propios es inaceptable si él ha sostenido la tesis de que el sentido de un nombre propio es idéntico al sentido de una descripción definida y, por tanto, es expresado mediante una descripción definida. La cuestión que cabe plantear es si Frege realmente ha sostenido esa tesis.

Hay autores, entre los que destaca Michael Dummett, que rechazan que quepa atribuir esa tesis a Frege. Dummett indica que el sentido de un nombre proporciona un criterio para identificar o reconocer un objeto como el referente del nombre y, aunque Frege admita que el sentido de un nombre puede ser idéntico al de una descripción definida, Dummett considera que de ninguna de las afirmaciones de Frege se sigue que él haya sostenido que el sentido de un nombre sea siempre el de una descripción definida. Dummett estima que al intentar decir cuál es el sentido de un nombre propio Frege se vio llevado de manera natural a indicar una descripción definida, pero de aquí no cabe inferir que el sentido de un nombre propio sea siempre idéntico al sentido de una descripción definida (véase Dummett 1981, pp. 97 s.). En otro lugar Dummett afirma que para que un hablante entienda un nombre propio se requiere que tenga la capacidad de identificar o reconocer el objeto que constituye el referente del nombre en presencia del objeto, pero no se requiere que haya de poder especificar cómo reconoce el objeto en cuestión; ahora bien, la captación del sentido de un nombre, al menos en el caso de algunos nombres propios, puede consistir simplemente en la asociación del nombre con dicha capacidad (Dummett 1978, p. 129).

En la misma línea de Dummett se encuentran otros autores, como Peter Geach y John Searle. Geach también rechaza que Frege haya sostenido la tesis de que el sentido de un nombre propio venga expresado mediante una descripción definida. Según Geach, no podemos identificar, reidentificar o discriminar los sentidos de los nombres propios mediante palabras, lo que no conduce, en opinión de Geach, a que los sentidos se tornen en entidades misteriosas y no-identificables. Geach establece un símil entre los sentidos de los nombres propios y las voces humanas:

[L]a mayoría de nosotros podemos identificar, reidentificar y discriminar voces humanas, pero no podríamos poner en palabras en qué consiste la individualidad de una voz dada; lo mismo ocurre con los sentidos de los nombres. (1980, p. 87). 
Por su parte, Searle, quien sostiene una teoría fregeana de la referencia de los nombres propios, ${ }^{9}$ afirma:

$[\mathrm{N}] \mathrm{o}$ todos los nombres propios paradigmáticos son iguales con respecto a la naturaleza de su 'contenido descriptivo'. Habrá, por ejemplo, una diferencia entre los nombres de personas vivas, con respecto a los cuales la capacidad del usuario del nombre para reconocer a la persona puede ser una importante 'descripción identificadora' [que el usuario asocia con el nombre de dicha persona - LFM], y los nombres de personajes históricos. (Searle 1969, pp. 173-174).

Por supuesto, en este pasaje la expresión "descripción identificadora” está siendo empleada en un sentido sumamente laxo, pero es digno de mención que Searle acepta que la capacidad de reconocimiento perceptivo de personas $-\mathrm{y}$, por tanto, de objetos, en la terminología de Frege- puede estar involucrada en la determinación de la referencia de los nombres propios de personas con quienes hemos estado en contacto perceptivo $-\mathrm{y}$ con quienes estamos suficientemente familiarizados-, y esa capacidad involucrará la discriminación de ciertos rasgos perceptibles, pero posiblemente muy complejos como para ser especificados lingüísticamente, al menos con facilidad.

Obviamente, si el sentido de un nombre propio puede consistir en una capacidad o en un criterio no-lingüístico para identificar o reconocer el referente del nombre, evitaríamos las consecuencias inaceptables que se seguían de la identificación del sentido de un nombre propio con el de una descripción definida y del requerimiento subsiguiente de que el sentido de un nombre propio viniese expresado mediante una descripción definida.

No obstante, la propuesta de Dummett, así como la de Geach y Searle, parece estar sujeta, al menos, a una importante limitación, a saber, los criterios no-lingüísticos para identificar o reconocer objetos como referentes de nombres no pueden ser considerados como los sentidos de todos los nombres -como Dummett y Searle explícitamente conceden-, pues hay objetos con los que nunca hemos estado en contacto perceptivo y con los que probablemente nunca lo estemos y que, por tanto, sólo podemos identificar lingüísticamente; piénsese, por ejemplo, en los nombres de personajes históricos, a los que Searle alude en el texto citado. Por este motivo, aunque Frege no haya sostenido expresamente que el sentido de cada nombre propio sea idéntico al sentido de una descripción definida y, por tanto, expresable mediante ella, en el marco de la posición de

9 Searle prefiere, en ocasiones (por ejemplo, en 1969, p. 170), afirmar que su teoría acerca de los nombres propios constituye un compromiso entre la teoría de Mill y la de Frege, pero su teoría es fundamentalmente fregeana, como él reconoce en (1983); véase, por ejemplo, 1983, pp. 197 s. 
Frege la única manera de hacer explícito de manera general y de comunicar el sentido de un nombre propio parece requerir la apelación a alguna descripción definida. Y de aquí se seguiría la tesis de que la referencia de un nombre propio viene determinada mediante una descripción definida, si bien esta descripción podrá ser distinta para hablantes diferentes. Ésta es, cuando menos, la tesis que Frege habría de sostener acerca de la referencia de los nombres propios que designan objetos con los que un hablante no ha estado en contacto perceptivo y que, por tanto, sólo puede identificar lingüísticamente.

De acuerdo con lo anterior, la contribución de un nombre propio a lo representado por el tipo de enunciados simples que estamos tomando en consideración -y, en general, por cualesquiera enunciados en los que el nombre figura-, especialmente en caso de que estos enunciados no versen acerca de objetos con los que el hablante ha estado en contacto perceptivo, es el objeto especificado por la descripción definida que el hablante asocia con el nombre propio, es decir, el objeto singularizado por ser el único que cae bajo el concepto designado por el término conceptual que figura en dicha descripción. Y si este término conceptual no se aplicase a un único objeto y, por tanto, la descripción no fuese satisfecha por un único objeto, el nombre en cuestión carecería de referencia, de lo que se seguiría que no lleva a cabo ninguna contribución a lo representado por los enunciados en los que figura o, mejor dicho, los enunciados en cuestión no representarían nada. Por lo que respecta al tipo de enunciados simples de los que nos estamos ocupando, lo representado por esos enunciados sería una relación entre un objeto y un concepto, pero en el caso mencionado no disponemos del objeto que pudiese estar en una relación con un concepto.

\section{EL SENTIDO Y LA REFERENCIA DE LOS TÉRMINOS CONCEPTUALES}

La tesis de que el sentido de un nombre propio es idéntico al sentido de una descripción definida es equivalente a la afirmación de que el nombre es definible mediante la descripción en cuestión. Ahora bien, puesto que cada descripción definida puede considerarse como formada a partir de un predicado monádico o término conceptual, simple o compuesto, es el momento de atender a la teoría de Frege acerca de los términos conceptuales.

Como ya indicamos anteriormente, Frege no nos ha dicho mucho acerca del sentido de estos términos, aunque cabe suponer que sus tesis al respecto serán, al menos en parte -aunque como indicaremos a continuación sólo en parte-, similares a las sostenidas acerca del sentido de los nombres propios. El sentido de un término conceptual determina el concepto que constituye la referencia del término, al proporcionar la condición que ha de ser satisfecha por dicho concepto. En el caso de los nombres propios ya hemos indicado que 
la interpretación más plausible de la posición de Frege, al menos por lo que concierne a los nombres de objetos con los que no hemos estado en contacto perceptivo, es que él ha sostenido que la referencia de un nombre propio viene determinada mediante una descripción definida -ésta puede ser diferente para hablantes diferentes-, que expresaría el sentido del nombre y, por tanto, ésta puede considerarse como proporcionando la definición del nombre. Por lo que concierne a los términos conceptuales cabría pensar que una tesis similar podría aplicarse, al menos, a los términos conceptuales que designan conceptos bajo los que caen objetos no perceptibles sensorialmente, y efectivamente los conceptos que a Frege le interesaron especialmente eran de este tipo, es decir, conceptos lógicos o lógico-matemáticos. Ahora bien, Frege no sostuvo, ni podría sostener, la tesis de que la referencia de un término conceptual venga determinada mediante una descripción definida, que expresaría el sentido del término. Tanto los nombres propios como las descripciones definidas son términos singulares, mientras que un término conceptual es un término general; por este motivo, el sentido de un término conceptual no puede venir expresado mediante una descripción definida, y ésta no puede determinar la referencia del término. ${ }^{10}$

En cualquier caso, Frege ha sostenido una tesis un tanto similar con respecto a los términos conceptuales que designan conceptos compuestos, es decir, conceptos que están compuestos de otros conceptos. La referencia de un término conceptual que tiene como referencia un concepto compuesto viene determinada por una definición -si bien ésta no adopta la forma de una descripción definida-en la que figuran los términos conceptuales que designan los conceptos componentes, la conjunción de los cuales constituye el definiens del término y expresa su sentido. ${ }^{11}$ El concepto en cuestión está con cada uno de sus conceptos componentes en la relación de subordinación, ${ }^{12}$ y de estos conceptos se dice que son características del primero. De esta manera, cuando un objeto cae bajo un concepto compuesto, cae también bajo los conceptos que constituyen sus características y tanto aquél como éstos son propiedades del objeto. ${ }^{13}$

10 A este respecto son famosas las consideraciones de Frege en $1892 \mathrm{~b}$-véase, por ejemplo, 1892b, p. 195- acerca de que una expresión que comienza con el artículo determinado en singular, como es el caso de las descripciones definidas, no puede designar un concepto y, por tanto, tampoco puede expresar el sentido de un término conceptual.

11 Un ejemplo puesto por Frege -véase 1892b, p. 202- es el siguiente. El concepto de ser un número entero positivo menor de 10 es definido mediante los conceptos de ser un número entero, ser un número positivo y ser menor de 10.

12 Un concepto está subordinado a otro concepto cuando todo objeto que cae bajo el primer concepto cae también bajo el segundo concepto.

13 Acerca de la distinción entre las nociones de característica y de propiedad en Frege véase, por ejemplo, 1884, sección 53. 
No obstante, no todos los términos conceptuales designan conceptos compuestos, por lo cual no todos los términos conceptuales pueden ser definidos. Tomando en consideración lo que Frege señaló en repetidas ocasiones con respecto a las entidades de carácter simple y a las expresiones que las designan, habrá términos conceptuales cuya explicación no pueda llevarse a cabo mediante una definición, sino que habremos de recurrir a elucidaciones (véase, por ejemplo, 1983, p. 224); en la elucidación de un término hacemos comprensible lo que se entiende por él -es decir, su sentido y, por ende, su referencia- recurriendo al empleo de expresiones del lenguaje natural, a pesar de que el uso de éstas no sea suficientemente preciso y esté sujeto a fluctuaciones, contando al respecto con la buena disposición a comprender por parte de la audiencia.

Llegados a este punto conviene traer a colación dos tesis de la teoría de Frege acerca de la referencia de los términos conceptuales que son especialmente relevantes para nuestros fines. Una de ellas es que Frege sostiene que hay términos conceptuales sin referencia, pero indica al respecto:

[É]stos no son los que unen lo contradictorio - pues un concepto bien puede ser vacío-, sino aquellos cuya delimitación es confusa. Para todo objeto debe estar determinado si cae o no bajo el concepto; un término conceptual que no satisfaga este requisito acerca de su referencia carece de referencia. (Frege 1892-1895, p. 133).

Por tanto, Frege sostiene la tesis de que los términos conceptuales carentes de referencia son aquellos que son vagos. Al menos dos consecuencias de esta tesis son dignas de mención. En primer lugar, de aquí se sigue que los enunciados que contengan este tipo de términos conceptuales no representarán nada. En el caso del tipo de enunciados simples que estamos tomando en consideración, la ausencia de su capacidad representacional se deberá a que el término conceptual correspondiente no nos proporciona un concepto bajo el cual pueda caer el objeto designado por el nombre propio que figura en el enunciado. Como indicamos anteriormente, sirviéndonos de la noción de propiedad, tales enunciados expresarían la atribución a un objeto de una propiedad, pero en este caso, según Frege, no hay ninguna propiedad designada y, por tanto, ninguna propiedad que pueda ser correcta o incorrectamente atribuida. Ahora bien, en nuestro discurso cotidiano empleamos enunciados que contienen términos conceptuales vagos, como "calvo", "árbol", etc. y consideramos que los enunciados que contienen estos términos pueden representar aspectos del mundo. En segundo lugar, las descripciones definidas en las que figuren términos conceptuales del tipo mencionado carecerán de referencia, con lo cual se ve reducido considerablemente el ámbito de las descripciones que podrán expresar el sentido de los nombres propios y determinar su referencia. 
La otra tesis de Frege acerca de la referencia de los términos conceptuales particularmente importante para el tema que nos ocupa es expresada por Frege de la siguiente manera:

Aquello a lo que se refieren dos términos conceptuales es lo mismo si y sólo si las extensiones de concepto correspondientes coinciden. (1892-1895, p. 133).

Dicho de otra manera, por lo que concierne a la referencia de los términos conceptuales dos conceptos son idénticos si tienen la misma extensión, es decir, si todo objeto que cae bajo uno de ellos cae bajo el otro, y viceversa. Pero de aquí se sigue, por ejemplo, que términos conceptuales como "criatura con corazón" y "criatura con riñones", cuyos conceptos poseen la misma extensión -o así podemos suponer-, designan el mismo concepto y, por tanto, que los enunciados "Juan es una criatura con corazón" -entendido en sentido literal- y "Juan es una criatura con riñones" habrían de representar lo mismo. Pero, aunque estos dos enunciados tengan el mismo valor de verdad, lo representado por ellos es intuitivamente $-\mathrm{y}$ a este respecto apelo a las intuiciones del lector-diferente; el primero representa que Juan tiene la propiedad de ser una criatura con corazón, mientras que el segundo representa que Juan tiene la propiedad de ser una criatura con riñones. Con objeto de dar cuenta de la diferencia en lo representado por esos enunciados y del hecho de que tales enunciados constituyen representaciones diferentes cabe sugerir los dos procedimientos siguientes.

Por una parte, podría alegarse que esas diferencias se deben a que cuando entendemos los dos enunciados mencionados captamos algo diferente, es decir, el pensamiento expresado por esos dos enunciados es diferente, y esto es así porque el sentido de los términos conceptuales "criatura con corazón" y "criatura con riñones" es diferente. Por este motivo, un proceder a seguir para explicar la diferencia en lo representado por ambos enunciados y, por ende, el hecho de que estos enunciados constituyen representaciones diferentes consistiría en dar un mayor papel en una teoría de la representación lingüística a la noción de sentido. Sin embargo, esta propuesta parece entrar en conflicto con una teoría puramente extensional de la representación lingüística, como la de Frege. En esta teoría, una vez que el sentido de una expresión, nombre propio o término conceptual, ha determinado una referencia, es decir, un objeto o un concepto, y este concepto, a su vez, una extensión, el sentido de la expresión desaparece de la escena y nos deja en su lugar sólo objetos, conceptos y extensiones de conceptos.

Por otra parte, es digno de mención que, aunque los términos conceptuales "criatura con corazón" y "criatura con riñones" designen conceptos con las mismas extensiones, constan de términos conceptuales, a saber, "corazón" y "riñón", que designan conceptos diferentes, pues las extensiones de estos dos 
conceptos son diferentes. Esta observación sugiere la siguiente modificación en el criterio de identidad de conceptos propuesto por Frege. Cabe conceder que conceptos simples son idénticos si sus extensiones son idénticas y que el concepto compuesto designado por un término conceptual es el mismo que el designado por la conjunción de los términos conceptuales que figuran en su definición. No obstante, por lo que concierne a términos conceptuales que designan conceptos compuestos pero que no están entre ellos en la relación de definición, como ocurre con los términos conceptuales "criatura con corazón" y "criatura con riñones", cabe requerir que para que términos conceptuales de este tipo designen el mismo concepto ha de cumplirse no sólo que los conceptos designados por estos términos tengan la misma extensión, sino que también tengan idéntica extensión los conceptos designados por los términos conceptuales más simples componentes de dichos términos conceptuales. Puesto que los términos conceptuales "corazón" y "riñón" poseen extensiones diferentes designan conceptos diferentes, de lo que se sigue que, de acuerdo con el criterio de identidad de conceptos recién propuesto, los términos "criatura con corazón" y "criatura con riñones" designarán conceptos diferentes y lo representado por los enunciados "Juan es una criatura con corazón" y "Juan es una criatura con riñones" será también diferente.

\section{CONCLUSIÓN}

La conclusión a extraer de nuestras consideraciones precedentes es que la teoría de la representación lingüística contenida en la obra de Frege, y especialmente la que concierne a la representación mediante los enunciados simples que constan de un nombre propio y de un término conceptual, que se basa en su teoría del sentido y de la referencia de los nombres propios y de los términos conceptuales, contiene ciertas deficiencias.

Frege parece sostener la tesis de que la referencia de los nombres propios -y esto ocurre de manera especial con respecto a los nombres propios de objetos con los que no hemos estado en contacto perceptivo- viene determinada mediante una descripción definida, que expresa el sentido del nombre, pero ya hemos indicado algunas de las consecuencias difícilmente aceptables de esta tesis. Por otra parte, puesto que cada descripción definida está formada a partir de un término conceptual, la teoría del sentido y de la referencia de los nombres propios formulada por Frege nos conduce a su teoría del sentido y de la referencia de los términos conceptuales.

Ahora bien, de la teoría de la referencia de Frege acerca de los términos conceptuales se siguen dos consecuencias recusables para una teoría de la representación lingüística. Una de estas consecuencias es que los enunciados que 
contienen términos conceptuales vagos, como lo son la mayoría de los términos conceptuales de los lenguajes naturales, no representan nada, de lo que se sigue que la mayoría de los enunciados de los lenguajes naturales no poseen el carácter de representaciones. En segundo lugar, otra consecuencia de dicha teoría es que términos conceptuales que designan conceptos que poseen la misma extensión llevan a cabo la misma contribución a la representación lingüística. Esta consecuencia entra en conflicto, al menos en algunos casos, como el anteriormente señalado, con nuestras intuiciones. A este respecto hemos propuesto dos procedimientos para evitar ese conflicto. Uno de ellos consiste en dar un mayor papel a la noción de sentido en una teoría de la representación lingüística; el otro radica en una modificación del criterio de identidad de conceptos formulado por Frege. ${ }^{14}$

\section{REFERENCIAS}

DUMMETT, M. (1978): Truth and Other Enigmas. Cambridge, Mass.: Harvard University Press.

DUMMETT, M. (1981): Frege. Philosophy of Language. Londres: Duckworth, $2^{\mathrm{a}}$ ed. rev.; $1^{\mathrm{a}}$ ed., 1973.

FERNÁNDEZ MORENO, L. (2006): La referencia de los nombres propios. Madrid: Trotta.

FREGE, G. (1884): Die Grundlagen der Arithmetik. Breslau: M. \& H. Marcus (reimp. en Hamburgo, Meiner, 1988).

FREGE, G. (1892a): "Über Sinn und Bedeutung”. Zeitschrift für Philosophie und philosophische Kritik, 100, pp. 25-50. Reimp. en G. Frege, Funktion, Begriff, Bedeutung, edición a cargo de G. Patzig, Gotinga, Vandenhoeck \& Ruprecht, 1962.

(Trad. cast. en Frege 1998).

FREGE, G. (1892b): "Über Begriff und Gegenstand". Vierteljahresschrift für wissenschaftliche Philosophie, 16, pp. 192-205. Reimp. en G. Frege, Funktion, Begriff, Bedeutung, edición a cargo de G. Patzig, Gotinga, Vandenhoeck \& Ruprecht, 1962. (Trad. cast. en Frege 1998).

FREGE, G. (1892-1895): “Ausführungen über Sinn und Bedeutung“, en Frege (1983), pp. 128-136. (Trad. cast. en Frege 1998).

FREGE, G. (1918): "Der Gedanke”. Beiträge zur Philosophie des deutschen Idealismus, 2, pp. 58-77. Reimp. en G. Frege, Logische Untersuchungen, edición a cargo de G. Patzig, Gotinga, Vandenhoeck \& Ruprecht, 1966. (Trad. cast. en Frege 1998).

14 La elaboración de este artículo ha contado con la financiación del proyecto FFI200803092 del Ministerio de Ciencia e Innovación. 
FREGE, G. (1976): Wissenschaftlicher Briefwechsel. Hamburgo: Felix Meiner.

FREGE, G. (1983): Nachgelassene Schriften. Hamburgo: Felix Meiner, $2^{\mathrm{a}}$ ed. rev. y ampliada; $1^{\mathrm{a}}$ ed., 1969.

FREGE, G. (1998): Ensayos de semántica y filosofía de la lógica. Madrid: Tecnos.

GEACH, P. (1980): "Some problems about the sense and reference of proper names", en F. Pelletier y C. Normore (eds.), New Essays in Philosophy of Language, Guelph, Canadian Association for Publishing in Philosophy, pp. 83-98.

KRIPKE, S. (1980): Naming and Necessity. Oxford: Blackwell. Reimp. revisada y con prefacio añadido de "Naming and necessity", en D. Davidson y G. Harman (eds.), Semantics of Natural Language, Dordrecht, Reidel, 1972. (Trad. cast.: El nombrar y la necesidad, México, UNAM, 1985).

SEARLE, J. (1969): Speech Acts. An Essay in the Philosophy of Language. Cambridge: Cambridge University Press. (Trad. cast.: Actos de habla: ensayo de filosofía del lenguaje, Madrid, Cátedra, 1980).

SEARLE, J. (1983): Intentionality. An Essay in the Philosophy of Mind. Cambridge: Cambridge University Press. (Trad. cast.: Intencionalidad: un ensayo en la filosofía de la mente, Madrid, Tecnos, 1992).

WITTGENSTEIN, L. (1922): Tractatus logico-philosophicus. Londres: Routledge \& Kegan Paul. (Trad. cast.: Tractatus logico-philosophicus Madrid, Tecnos, 2003, $2^{\text {a }}$ ed. rev.; $1^{\mathrm{a}}$ ed. 2002). 